Natur und Wissenskulturen 
Hans Jörg Sandkühler

\title{
Natur und Wissenskulturen
}

\author{
Sorbonne-Vorlesungen \\ über Epistemologie und Pluralismus
}

Verlag J.B. Metzler

Stuttgart - Weimar 
Die Deutsche Bibliothek -CIP-Einheitsaufnahme

Ein Titeldatensatz für diese Publikation ist bei der Deutschen Bibliothek erhältlich.

ISBN 978-3-476-01920-2

ISBN 978-3-476-02899-0 (eBook)

DOI 10.1007/ 978-3-476-02899-0

Dieses Werk einschließlich aller seiner Teile ist urheberrechtlich geschützt. Jede Verwertung außerhalb der engen Grenzen des Urheberrechtsgesetzes ist ohne Zustimmung des. Verlages unzulässig und strafbar. Das gilt insbesondere für Vervielfältigungen, Übersetzungen, Mikroverfilmungen und die Einspeicherung und Verarbeitung in elektronischen Systemen.

(C) 2002 Springer-Verlag GmbH Deutschland

Ursprünglich erschienen bei J.B. Metzlersche Verlagsbuchhandlung und Carl Ernst Poeschel Verlag GmbH in Stuttgart 2002

www.metzlerverlag.de info@metzlerverlag.de 


\section{Inhalt}

Vorbemerkung

1. Natur und Wissenskulturen. Zur Einführung 12

1.1 Warum mit Kant beginnen? 13

1.2 Die Auflösung des Gegebenen und die pluralistische Idee von Welten 15

1.3 Die Wahrheit gewisser Erfahrungssätze gehört zu unserm Bezugssystem: Begriffs-Relativität und epistemologische Profile

1.4 Die Fragwürdigkeit des Konzepts ,Repräsentation' 25

1.5 ,Sehen' statt ,repräsentieren' - konstruieren statt abbilden 31

1.6 ,Tatsachen' im interpretatorischen Raum: homo mensura auch in den Wissenschaften

2. Vom britischen Empirismus zu Kants Kopernikanischer Revolution in der Epistemologie $\quad 40$

2.1 Erfahrung, Rationalität und Interpretation der Natur 41

2.2 Kants ,Revolution der Denkungsart' 46

3. Naturerkenntnis und Gesellschaftstheorie. Die Idéologie als empirische Alternative in der Erkenntnistheorie und die ,Entdeckung einer neuen Wissenschaft der Gesellschaft'

3.1 Destutt de Tracy: Die Idéologie als empirische Wissenschaft von den Ideen

3.2 Saint-Simon: Der physicisme als , allgemeine Wissenschaft' und die Reorganisation der Gesellschaft

3.3 Fourier: Die Entdeckung einer neuen Wissenschaft 
4. „Wie die Naturwissenschaft den Idealismus aus dem Realismus hervorbringt." Zur Naturphilosophie Schellings

5. Die menschliche Natur, die Geschichte, das Recht und der Staat als ,zweite Natur'. Zu Schellings politischer Philosophie

5.1 Schelling - Theoretiker der Geschichtlichkeit aller Existenz

5.2 Die Naturnotwendigkeit von Recht und Staat

6. Die Physique sociale und die Naturgesetze der Gesellschaft

7. Tatsachen und Theorien. Die Krise des Positivismus und die Philosophie der induktiven Wissenschaften

7.1 William Whewell: Nature is the Book, and Man is the Interpreter

7.2 John Stuart Mill: Wie ist Verallgemeinerung aus Erfahrung möglich?

7.3 Friedrich Adolf Trendelenburg: Das Objektive ist keine ungemischte Nachricht von Eigenschaften der Dinge

8. Gesellschaftstheorie zwischen Philosophien und Wissenschaften: Marx' problematische Bezugnahme auf die Naturwissenschaften

8.1 Marx in Kontexten

8.2 ,Wirklichkeit' und ,Kritik' - eine problematische Allianz

8.3 Auf der Suche nach der verlorenen Wirklichkeit: Empirie statt Spekulation

8.4 Das Dilemma der substanzontologischen ,Dialektik'

9. Physiologie und Philosophie - Naturwissenschaften und das Bedürfnis nach Epistemologie im 19. Jahrhundert 
9.1 Johannes Müller: Physiologie auf philosophischer Grundlage

9.2 Hermann von Helmholtz: Die Warnung vor blinder Empirie

9.3 Emil du Bois-Reymond: Die Grenzen der Erkenntnis

9.4 Heinrich Hertz und Ludwig Boltzmann: „daß keine Theorie etwas Objektives sein kann“"

10. „Wir fangen mit dem Denken an." Der Neukantianismus oder: Zurück zu Kant und über Kant hinaus 159

10.1 Der Marburger Neukantianismus: Anschauung ist Denken

10.2 Der Heidelberger Neukantianismus: Die Wirklichkeit wird Natur, wenn ...

10.3 Kantische Motive und Prinzipien außerhalb des deutschen Neukantianismus

10.3.1 Husserl: Der Widersinn einer, naturwissenschaftlichen Erkenntnistheorie'

10.3.2 Peirce: Alles Denken ist ein Denken in Zeichen

11. Die ,Natur' des Rechts und der Relativismus im Recht

11.1 Pluralismus, Relativismus und die Geltung des Rechts

11.2 Die ,Natur' des Rechts. Kausalität oder Normativität?

11.2.1 Naturrecht

11.2.2 Rechtspositivismus

11.3 Sein und Sollen; Kausalität, Normativität und Zurechnung

11.4 Relativismus und Recht

11.4.1 Kelsen: Trennung von Recht und Moral, Recht und Gerechtigkeit

11.4.2 Radbruch: Recht ist die Wirklichkeit, die den Sinn hat, der Rechtsidee zu dienen 
12. Wissenskulturen - Theorie der Erkenntnis, des Wissens und der Wissenschaft ex analogia hominis. Cassirer und der neue wissenschaftliche Geist

12.1 Erkenntnistheorie im Übergang von der Kritik der Vernunft zur Kritik der Kulturen der Erkenntnis: Sprache, Zeichen und Symbol

12.2 Ein neuer wissenschaftlicher Geist, oder: „Erkennen, daß alles Faktische schon Theorie ist" 230

12.3 Kulturkritik und Wissenschaftskritik

13. Der Rückfall in den Naturalismus: Realismus, Repräsentation und naturalisierte Epistemologie

13.1 Realismus: Die Welt, unabhängig vom Bewußtsein?

13.2 Repräsentation: Es keine magischen, unersetzbaren Verbindungen zwischen einer Sprache und der Welt

13.3 Mentale Repräsentation 260

13.4 Naturalismus in der Philosophie des Geistes 264

13.4.1 Merkmale des metaphysischen Naturalismus 264

13.4.2 Aspekte einer Kritik am metaphysischen Naturalismus

14. Welt-Versionen

14.1 Nelson Goodman: The world is in many ways 272

14.2 Hilary Putnam: Objectivity and rationality humanly speaking

Bibliographie

Personenregister 


\section{Vorbemerkung}

In den Studien, die ich zur Epistemologie, zur Wissenschaftsphilosophie und zur Wissenschafts- und Philosophiegeschichte im 19. und 20. Jahrhundert vorlege, geht es um das Sprechen über die Natur, nicht um Dialoge mit der Natur. Die Geschichte des Sprechens über die Natur Geschichte von den Mythen und Religionen, Künsten, Wissenschaften und Philosophien - zeigt, daß die Natur nicht selbst und nicht auf ,natürliche' Weise spricht. Die Frage, was Natur ist, übersetze ich deshalb in zwei Problemstellungen: Wie verständigen wir uns über die Natur, wenn es nicht sie ist, die zu uns spricht? Wie wird in Philosophie und Wissenschaften über die Natur gesprochen? Und: Was sind die Gründe dafür, daß über die Natur in so verschiedenen Sprachen, in so verschiedenen Perspektiven, in so verschiedenen Theorien so unterschiedlich gesprochen werden kann?

Ich behandle die Natur in Sprachen der Kultur nicht in der Perspektive der Naturphilosophie, sondern in jener der Epistemologie. Mein Interesse gilt systematischen Problemen, doch ich werde die Probleme in historischen Kontexten erläutern und in Konstellationen, in denen die Beziehung zwischen Philosophie und Naturwissenschaften auf der Tagesordnung steht - eine, wie zu sehen sein wird, problematische Beziehung. Soweit das Problem ,Natur' dort von Belang ist, beziehe ich mich auch auf die Gesellschafts- und Rechtstheorie.

Das Problemfeld ist die theoretische Philosophie, näher: Epistemologie und Wissenschaftsphilosophie. Mein Ziel liegt jenseits der theoretischen Philosophie. Ich versuche, die epistemische condition humaine zu beschreiben, in der Menschen heute denken und handeln denken und handeln könnten. Um es auf einen Nenner zu bringen: Ich möchte zeigen, wie der Pluralismus und die mit ihm verbundene Anerkennung der Relativität von Überzeugungen und Wahrheiten aus guten Gründen entstanden sind und warum ich sie für eine angemessene philosophische Einstellung zur modernen Welt für konstitutiv halte.

Ich habe diesen epistemologischen Pluralismus nicht immer vertreten. Auf der Grundlage eines an Marx orientierten Verständnisses habe ich noch Mitte der 1980er Jahre gegen den relativistischen Plural argumentiert. Nicht weniger nachdrücklich habe ich mich gegen jeden als die eine Wahrheit verkleideten dogmatischen Anspruch gewandt. Mit 
den Theorien gearbeitet zu haben, die mit dem Namen ,Marx' verbunden sind, war sinnvoll; politische Schlußfolgerungen, die ich aus ihnen gezogen habe, gehören zu den Fehlern, die ich zu verantworten habe. Meine ,idealistische Wende' in der Theorie wurde, Jahre vor 1989, von Marxisten kritisiert, die in mir den Kantianer entdeckt hatten. Die Kritik hat sich als hilfreiche Therapie zum Pluralismus erwiesen. Wer viele ,Wahrheiten' ausprobiert hat, wird bescheidener, tolerant, pluralistisch. Überzeugungen, Meinungen und Hoffnungen sind vorläufig; dies ist meine philosophische und politische Zwischenbilanz. Der Pluralismus ist allerdings auch eine problematische Herausforderung; in seinem Schatten nistet ein Relativismus, der nicht zu akzeptieren ist. Menschenwürde und Menschenrechte sind nicht relativierbar. Ohne Pluralismus sind sie aber nicht denkbar. ${ }^{1}$

Der faktische Pluralismus demokratischer Gesellschaften ist für mich zum zentralen Thema geworden. Es hat scheinbar nur eine politische Dimension. Doch grundlegend für die Demokratie ist die Dimension von Wissen und - vor allem - Urteilsfähigkeit. Der epistemische Pluralismus hat mich zunächst als Erkenntnistheoretiker beschäftigt. In der zweiten Hälfte der 1980er Jahre hat mich meine Kritik an materialistischen Widerspiegelungstheorien an den Punkt geführt, an dem ich für erneute und für neue Lektüren offen war. Noch deutlicher als zuvor an Kant orientiert, habe ich für mich die Epistémologie Bachelards entdeckt, den Pragmatizismus von Peirce und die Analytische Philosophie in der Phase ihrer Erneuerung durch die Kantische Kritik, wie sie nicht zuletzt von Goodman und Putnam reformuliert worden ist. Diese Philosophien geben gute Gründe dafür an, die Kontextualität und Perspektivität des Erkennens und Wissens und deren Vernetzung mit Sprachen, Theorierahmen, Begriffsschemata und historischen Formen der Praxis ernst $\mathrm{zu}$ nehmen und philosophisch zu analysieren. Niemand hat einen archimedischen Standpunkt; statt dessen wählen wir Perspektiven aus Möglichkeitsfeldern; wir wählen epistemologische Profile. Diese Einsicht hat mich zu Cassirers Philosophie der symbolischen Formen und zu einer kritischen Epistemologie in Nähe zu den Wissenschaften und zu anderen symbolischen Kulturen geführt.

Mein Bericht zu Selbstverständigungen über die Natur und über die Geschichte des Pluralismus als Geschichte des Zerfalls vormaliger Evidenzen entspricht der Wahl eines epistemologischen Profils. Es be- 
gründet meine Sicht auf eine Geschichte, für die ich interessieren möchte, ohne dabei zu verkennen, daß ein anderer roter Faden - z.B. jener der Oppositionen gegen den Pluralismus - durchaus denkbar ist.

Die Antwort auf die Fragen, Was können wir wissen und wie sollen wir handeln?' setzt immer wieder ein bei einer Kritik, deren Prinzipien zunächst Kant benannt hat. Wer philosophiert, sollte sich ihr verpflichtet wissen; er wird dann den Pluralismus von Perspektiven nicht als Last hinnehmen, sondern wünschen. Ich erwähne zwei frühere Bücher, weil sie diesen Wunsch signalisiert haben: Die Wirklichkeit des Wissens. Geschichtliche Einführung in die Epistemologie und Theorie der Erkenntnis (1991) und Demokratie des Wissens. Aufklärung, Rationalität, Menschenrechte und die Notwendigkeit des Möglichen (1991). Sie gründen in der Überzeugung, da $ß$ wir selber es sind, die im Erkennen Welten entwerfen, unter denen es die allein wahre nicht gibt. Wenn dies so ist, dann ist der Pluralismus der Erkenntnis-Welten Bedingung der Ordnung des Rechts, in der alle ihre Freiheit finden.

Für mich wichtige Impulse habe ich durch die Beschäftigung mit ausgewählten Kapiteln der Wissenschaftsgeschichte gewonnen, vor allem aber durch interdisziplinäre Forschungen zur Wissenschaftsphilosophie z..B. der Neurowissenschaften und der Rechts- und Staatswissenschaft. Besondere Anregungen verdanke ich der Arbeit im Bremer Zentrum Philosophische Grundlagen der Wissenschaften.

Den Anlaß, Forschungsergebnisse zu einem Ganzen zu bündeln ${ }^{2}$, haben meine Vorlesungen an der Université Paris 1 Panthéon-Sorbon$n e$ geboten, gehalten im Frühjahr 2001 im Rahmen einer Gastprofessur. Prof. Jean-François Kervegan danke ich herzlich für seine Einladung.

Bremen, im November 2001

H.J.S.

2 In dieses Buch sind - überarbeitet - publizierte Vorarbeiten eingegangen. Auf einige verweise ich in Fußnoten. Zur Bibliographie weiterer Arbeiten vgl. Herausforderung Pluralismus. FS für Hans Jörg Sandkuihler. Hg. v. M. Plümacher, V. Schürmann und S. Freudenberger, Frankfurt a.M. Zu einer Einführung in die Epistemologie, auf die ich hier gelegentlich zurïckgreife, vgl. Sandkühler 1991. 